\title{
Representaciones sociales en el proceso de capacitación en directivos y empleados de varios sectores económicos en \\ Medellín, Colombia
}

\author{
Social representations in training process directors and \\ employees in Medellín's, Colombia several economic sectors
}

Daimer Higuita-López

Universidad Nacional de Colombia, Medellín, Colombia.

\begin{abstract}
Resumen
Objetivo: Analizar las representaciones sociales que se construyen directivos y empleados en el proceso de capacitación. Método: investigación cualitativa en la que se aplicaron entrevistas a 18 directivos del área de gestión humana y a 33 trabajadores de varios sectores económicos en la ciudad de Medellín. Para la construcción de las representaciones, se utilizó la carta asociativa, cuya finalidad fue identificar las características de la capacitación. Resultados: se encontró que en la representación de los directivos, la capacitación es una estrategia que permite alinear al empleado y fortalecer aquellas habilidades que generen valor para la compañía. En cuanto a la representación de los trabajadores, la capacitación está asociada al conocimiento que reciben y al valor que sienten por parte de la organización al momento de capacitarlos. Discusiones: No es sencillo trazar una línea causal entre capacitación y rendimiento individual, como sugieren los textos de recursos humanos, porque las empresas que desarrollan capacitación evalúan solamente la percepción y el aprendizaje pero no evalúan la aplicación de conocimientos ni los resultados de la capacitación en términos de desempeño. Conclusiones: Entre trabajadores y directivos existe esa representación, es decir, esa idea de causalidad entre capacitación y rendimiento. Igualmente, la sola existencia de la capacitación es asumida como una muestra de que los trabajadores son importantes para las empresas.
\end{abstract}

Autor de Correspondencia

dhiguitalo@unal.edu.co

Recibido: 08-12-2019

Aceptado: 27-03-2020

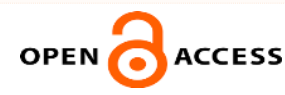

\section{(cc) BY}

Copyright (C) 2020

Desarrollo Gerencial
Palabras clave: Aprendizaje organizacional, desempeño laboral, mejora continua, sistemas cognitivos, valoración del personal.

Clasificación JEL: M12

\begin{abstract}
Purpose: To analyze the social representations produced by directors and employees during training process. Method: Qualitative research involving the administration of surveys to 18 human talent directors and 33 workers in different economic sectors in the City of Medellin. To create the representations, the associative card was used to identify the characteristics of the training. Results: The directors' representation of training is that it is a strategy used to align employees and enhance the skills that add value to the company. The workers' representation of training is related to the knowledge received and sense of security in their value to the company upon the training session. Discussion: It is not easy to establish a causal relationship between training and individual performance, as suggested by literature on human resources, because the companies that offer training only assess perception and learning but fail to evaluate the application of knowledge and training results in terms of performance. Conclusions: Workers and directors share such representation, i.e., the causal relationship between training and performance. Moreover, the mere existence of training is considered proof that companies care for their employees.
\end{abstract}

Keywords: Organizational learning, work performance, continuous improvement, cognitive systems, personnel appraisal.

JEL Classification: M12

Como citar este artículo (APA):

Higuita-López, D. (2020). Representaciones sociales sobre el proceso de capacitación en directivos y empleados de varios sectores económicos en Medellín, Colombia. Desarrollo Gerencial, 12(1), 1-20. https://doi.org/10.17081/dege.12.1.3757 


\section{Introducción}

Dentro de cualquier tipo de organización el área de gestión humana constituye un apoyo fundamental para el desarrollo de los procesos, por medio de ella se busca optimizar los resultados y mejorar las condiciones laborales de los trabajadores (Shaheen, Ghayyur y Yasmeen, 2014); una de estas formas es a traves de la capacitación que les ofrecen a los empleados (Kooij y Boon, 2018). Para las directivos de las organizaciones contemporáneas dentro de su misión y visión, el capital humano es lo más importante para la compañía (Tito, 2003) y se fortalece mediante bonificaciones (aspectos tangibles), ascensos, reconocimientos o capacitación (aspectos intangibles) (Rueda, Jiménez y Sánchez, 2015). De manera que mejorar las condiciones de los empleados a través de la capacitación es una de las formas más consistente de demostrar que el capital humano es importante, ya que tener equipos de trabajo capacitados y motivados con lleva al éxito y al cumplimiento de los objetivos organizacionales.

En este sentido, Mohamed y Mustafa (2018) señalan que por medio de la capacitación se busca incrementar o mejorar las habilidades técnicas o sociales de los trabajadores, aumentar su desempeño, y como lo afirma Young y Nam (2018) tiene efectos positivos en el compromiso, la productividad y la satisfacción laboral del capital humano. Sin embargo, esta debe ser evaluada revisando las percepciones de los capacitados, la aplicación de lo aprendido y el impacto que ha tenido en el desempeño de los empleados (Kooij y Boon, 2018). Ahora bien, el hecho de que la literatura académica insista en que la capacitación es muy benéfica para empleados y directivos, pero poco o nada apunte a los efectos adversos de esta, genera dudas. Y una primera duda gira alrededor de si efectivamente la capacitación tiene tales impactos o sencillamente se ha construido una representación social como tal.

Como se explicará más adelante, las representaciones sociales implican creencias, estereotipos y normas que suelen tener una actitud positiva o negativa (Araya, 2002) y una reacción emocional sobre algo. Además, pueden orientar el actuar de una persona ya que implica principios interpretativos a través de la comprensión, valoración y actuación. En este sentido, surgen preguntas como: ċlos efectos positivos de la capacitación existen en el imaginario colectivo de trabajadores y directivos? ¿Qué representaciones sociales se configuran en el entorno empresarial sobre la capacitación? En consecuencia, este artículo se propuso analizar las representaciones sociales que se construyen directivos y empleados acerca del proceso de capacitación.

No obstante, antes de entrar en detalle sobre el presente estudio, es importante indicar algunas precisiones: esta investigación no buscó medir el impacto de la capacitación en el desempeño ni validar la tesis sobre si la capacitación aumenta la productividad organizacional o no, aunque la revisión de la literatura sugiera que sí, el propósito fundamental de este estudio fue conocer qué representaciones 


\section{Daimer Higuita López}

sobre la capacitación tienen directivos y empleados, en algunas empresas de Medellín. Asimismo, teniendo en cuenta la importancia del capital humano, se indagó si solo la capacitación contribuye con la construcción de la representación en los trabajadores, es decir, esto implica determinar que aunque hay empleados que no han sido capacitados, estos tienen el conocimiento que la organización brinda capacitación y por lo tanto se constituyen como un componente importante que deben ser motivados y entrenados para buen el funcionamiento y el éxito organizacional.

Como segunda precisión, el estudio no tiene pretensiones de generalizar los resultados a otras organizaciones, básicamente se entrevistó un grupo de directivos de gestión humana y un grupo de empleados de organizaciones distintas a las de los directivos-como se explicará en la sección de la metodología-luego entonces, este artículo parte de una breve revisión de la literatura, aborda las nociones de capacitación, formación, aprendizaje y representaciones. Seguidamente, describe el proceso metodológico sobre el trabajo de campo, el cual se caracteriza por tener un enfoque cualitativo. Posterior a ello, se presentan los hallazgos encontrados en términos de las representaciones para los directivos y trabajadores y finalmente se aportan las conclusiones de la investigación.

\section{Fundamentación teórica}

Actualmente en el ámbito empresarial es bastante común hablar del impacto que tiene la capacitación en los empleados, autores como Bercu (2017) señalan que los programas de capacitación ayudan a la formación y satisfacción del empleado; Vutâ y Farcâs (2015) sostienen que identificar las necesidades de capacitación de los empleados, aumenta la productividad de la empresa. Rueda, Jiménez, y Sánchez (2015) indican que existe una relación positiva entre la efectividad de los proyectos de la organización y los programas de capacitación. Asimismo, Landini, Bianqui, y Russo (2013), evalúan tanto la implementación como el impacto de la capacitación en las creencias de los extensionistas. En general, manifiesta que la capacitación de los trabajadores lleva a la empresa a obtener mejor calidad y productividad (Gil y Mataveli, 2016). Se argumenta que la capacitación es una excelente forma para que la empresa esté preparada para los cambios venideros. Para Mohamed y Mustafa (2018), los efectos positivos de la capacitación sobre el desempeño están asociados particularmente a un método que se ajusta a la especificidad de cada organización.

En el campo organizacional, el tema de las representaciones ha sido estudiado porque contribuyen con una identidad social y la búsqueda de sentidos (Araya, 2002), su estudio ha estado vinculado con categorías como cultura organizacional, compromiso e identidad organizacional. Algunos de los autores que han abordado este tema son: Alvesson (2015); Alvesson y Willmott (2013); y Alvesson y Willmott (2014). Particularmente, Flouris y Kucuk (2017) destacan que integrar las prácticas de recursos humanos y los riesgos corporativos, moviliza las representaciones y ayuda a mejorar el rendimiento. Raigosa y 


\section{Daimer Higuita López}

Marín (2010) consideran la representación media que hay entre la percepción y los niveles de engagement en las organizaciones. Por otra parte autores como Esquivei, Félix y Bello (2014); Fawad (2010); Mazur y Woodland (2017); O 'Connor y Little (2012) abordan el impacto que tiene la capacitación. En el área de las humanidades, los principales autores sobre las representaciones son Moscovici (1961), Jodelet (1986); Morin (2016) y Abric (2001), este último estudia la recursividad entre las representaciones y las prácticas en enfermeras y agentes comerciales.

\section{La Nociones Capacitación, Aprendizaje y Representación}

La noción de capacitación se construyó con base en textos que son frecuentemente utilizados para la formación de profesionales en el campo de la gestión humana (Burbano, 2016). Algunos de estos textos son: La gestión de los recursos humanos de Dolan, Valle, Jackson y Schuler (2007); Administración de recursos humanos de Chiavenato (2009) y Administración de Recursos Humanos de Dessler (2009). Se acudió a estos textos porque, probablemente, con la forma como relatan el proceso y los beneficios de la capacitación, contribuyen con la construcción de la idea de que la capacitación es una manera de concretar el interés en el empleado.

La capacitación es entendida como el proceso que busca la adaptación y preparación a trabajadores, mediante la adquisición de conocimientos asociados a un proceso productivo. Sobre las etapas de la capacitación, los textos proponen una estructura similar: definen el concepto, explican el objetivo de la capacitación y establecen los pasos para diseñar un programa de capacitación. Esos pasos van desde identificar las necesidades de capacitación, el diseño del programa, su implementación y finalmente se evaluación. Para Mohamed y Mustafa (2018), son muy importante las representaciones que los trabajadores se hacen del capacitador. Estos autores sostienen que cuando los trabajadores tienen la idea que pueden aprender del capacitador, entonces los resultados parecen más favorables.

Usualmente, los cuatro aspectos que se recomienda evaluar son: la percepción sobre el curso de capacitación, el aprendizaje que esta generó, la aplicación en el lugar de trabajo de lo aprendido y los resultados en términos de mejoras en el desempeño. Algunas de las preguntas en el trabajo de campo indagaron si se evalúan estos cuatro aspectos. 
Figura 1. Evaluación de la capacitación recomendada en los libros de texto

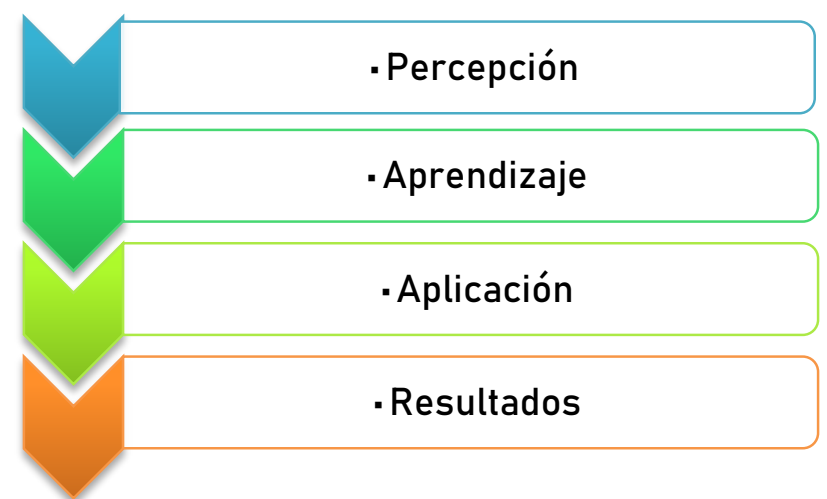

Fuente: Elaboración propia (2019).

Para la noción de aprendizaje se acudió a otras fuentes bibliográficas, y aunque existe una amplia literatura sobre ésta, en especial desde la psicología, aquí solamente se hará mención a dos posiciones sobre la adquisición de conocimiento. La escuela Racionalista propuso la razón como fuente de conocimiento; mientras que para el Empirismo, el aprendizaje se fundamenta en la experiencia (Jaraba, 2012). Ahora bien, si la capacitación genera un conocimiento-aprendizaje este podría obedecer tanto a un proceso racional del cerebro (durante y después de recibir la capacitación) como a una prácticaexperiencia, cuando la capacitación exige ir practicando lo "enseñado", es decir, no necesariamente tiene que existir una sola vía o una sola causa de aprendizaje, por ejemplo, Kooij y Boon (2018) sostienen que las percepciones sobre la capacitación mejoran las representaciones sobre el aprendizaje y esto, se cree, que impacta el compromiso.

Por su parte, las representaciones sociales se pueden definir como sistemas cognitivos que implican creencias, estereotipos, opiniones y normas, que suelen tener una actitud positiva o negativa (Araya, 2002). La actitud se vincula con la reacción emocional sobre un objeto o un hecho. Las representaciones sociales pueden entenderse, entonces, como formas de pensamiento del sentido común, o como significaciones que instituyen un orden (Morín, 2016). En otras palabras, la representación puede orientar el actuar de un individuo o un colectivo: socialmente se da una presión que reclama opiniones, posturas y acciones acerca de los hechos que están focalizados por el interés público. Las exigencias grupales para el conocimiento de determinado evento u objeto aumentan a medida que su relevancia crezca (Materán, 2008). Las representaciones incorporan principios interpretativos y orientadores de las prácticas, a través de cuatro funciones: comprensión, valoración, comunicación y actuación.

Las representaciones sociales se generan mediante objetivación y anclaje. La objetivación lleva a hacer real un esquema conceptual. La objetivación es una operación formadora de una imagen estructurante. 
Es el paso de un conocimiento científico al dominio público. Con el anclaje la representación social se liga con el marco de referencia de la colectividad y es un instrumento útil para interpretar la realidad y actuar sobre ella. El anclaje implica la integración cognitiva del objeto de representación dentro del sistema preexistente del pensamiento y sus respectivas transformaciones (Materán, 2008).

Cuando se expone sobre contenido de una representación se alude a una forma particular de conocimiento, que constituye un universo de creencias. Conocer una representación social implica determinar qué se sabe (información), qué se cree, cómo se interpreta (campo de la representación) y cómo se actúa (actitud) a partir de esa idea o creencia. De acuerdo con Moscovici, citado en Araya (2002) "estas tres dimensiones constituyen un conjunto que tan sólo puede escindirse para satisfacer las exigencias propias del análisis conceptual" (p. 41). Precisamente, advertimos al lector que el apartado Hallazgos se ha "escindido" en directivos y trabajadores para facilitar el análisis. Pero es importante indicar que las estructuras y los elementos nucleares de las representaciones de directivos y empleados pueden estar sumamente conectados. Las Figuras 3 y 4 muestran que estas dos poblaciones tienen representaciones similares frente a aspectos como el desempeño organizacional.

Young y Nam (2018) indican que hay efecto positivo indirecto de la capacitación sobre el desempeño, ya que el compromiso puede hacerse más fuerte. Pero, hay dos variables importantes: la voluntariedad y la no evaluación. Estos autores afirman que cuando los empleados tienen la representación de que es obligatorio o que su aprendizaje será evaluado, los efectos sobre el desempeño parecen ser menores. Por lo tanto, en la evaluación que se haga de la capacitación es pertinente considerar las representaciones que se hacen los sujetos sobre dicha capacitación. Y este fenómeno es el objeto del presente documento.

En este punto es pertinente reiterar que la investigación buscaba analizar las representaciones sociales que se construyen directivos y empleados acerca del proceso de capacitación, e indagar si la sola existencia de la capacitación contribuye con la representación en los trabajadores sobre si ellos son importantes para la organización. De allí que sea necesario, como se verá en el siguiente apartado, entrevistar a empleados que no han recibido capacitación. En breve: si se encuentran casos de empleados que no han recibido capacitación y consideran que la capacitación ofrecida es algo que les mejora las condiciones, podría pensarse que en el entorno organizacional la sola existencia de la capacitación contribuye con la representación social de que los trabajadores son importantes.

\section{Método}

\section{El Trabajo sobre el Terreno: Diseño, Participantes e Instrumentos}

Para el análisis de las representaciones sobre la capacitación, el estudio se apoyó en la investigación cualitativa, ya que "el sentido de la vida social se expresa a través de discursos que emergen en la vida 


\section{Daimer Higuita López}

diaria" (Guber, 2001, p. 33). En el trabajo de terreno se aplicaron dos instrumentos de entrevista semiestructurada: uno para 18 directivos de gestión humana y el otro para 33 empleados, de organizaciones distintas a las de los directivos. Se buscó que unos y otros pertenecieran a organizaciones distintas con un interés metodológico: evitar que durante la interpretación, de las representaciones de un empleado particular, se asumirá que una representación era propia de una organización en particular (orientada por el discurso del director de RH). La mayoría de los directivos sostienen haber participado en el diseño de cursos de capacitación o haber sido capacitados. En contraste, solo 20 de los 33 trabajadores habían recibido capacitación. Las entrevistas se realizaron en el lugar de trabajo y duraron entre 30 y 40 minutos cada una.

Los entrevistados pertenecen a distintos sectores económicos, desde servicios informáticos, establecimientos comerciales, hasta empresas de transporte. En cuanto a características de género, edad, área en la empresa o nivel académico, puede expresarse que las dos poblaciones son bastante heterogéneas.

En el análisis se utilizó la carta asociativa, para rastrear los rasgos más característicos de lo que representa la capacitación. Esta metodología se elaboró siguiendo los procedimientos de Abric (2001), quien plantea diferentes técnicas para analizar las representaciones. Así, se tomaron inicialmente cuatro entrevistas de directivos y se diseñó un esquema con las posibles categorías emergentes. Para la nominación de estas categorías se analizaron expresiones utilizadas por los directivos, tales como valorar, desempeño, filosofía organizacional. En efecto, por las técnicas utilizadas en la investigación cualitativa, la información suele expresarse en cadenas verbales y no mediante valores numéricos (Araya, 2002). El boceto inicial se apoyó en las respuestas a preguntas como ¿por qué cree que la empresa brinda capacitación a los empleados?

Posterior a lo anterior, se revisaron las 14 entrevistas restantes de directivos, y se hicieron agrupaciones para consolidar las categorías emergentes, las cuales se pueden apreciar en la Figura 2. El mismo proceso se realizó con las entrevistas hechas a los empleados: primero se tomaron cuatro entrevistas, se armaron unas pre-categorías o esquemas de asociaciones y con estas se leyeron las restantes entrevistas de los trabajadores. El esquema con estas representaciones se expone en la Figura 5.

\section{Resultados y Discusiones}

Lo primero que es importante señalar son los hallazgos sobre la evaluación de la capacitación; de los cuatro elementos planteados se tiene que: en las 51 organizaciones, al finalizar la capacitación se evaluó la percepción. El aprendizaje se analizó en algunos casos (35), sobretodo en temas, por ejemplo, de 


\section{Daimer Higuita López}

seguridad industrial. Y no se encontró evidencia de la evaluación de la aplicación (de lo aprendido con la capacitación) ni de los resultados en términos de desempeño.

Como mencionó anteriormente, este apartado se ha organizado en dos secciones, de acuerdo con las dos poblaciones estudiadas. No obstante, veremos que las representaciones sociales (y los argumentos que las sostienen) parecen repetirse en la construcción de las estructuras de las representaciones de directivos y empleados.

\section{Las representaciones de los directivos}

Las nociones que tienen los directivos sobre el proceso de capacitación son, primordialmente, representaciones con una orientación positiva, fundamentadas en que la capacitación permite alinear a los trabajadores ya que mejora su desempeño y es una manera de mostrar interés por ellos. Y en los casos donde se avizora una orientación negativa, esta es dada porque la capacitación, según los directivos no contribuye a los objetivos organizacionales.

A continuación se describe primeramente la orientación positiva de las representaciones en los directivos:

La idea de la capacitación como medio para alinear emerge con fuerza porque los directivos creen que el empleado debe incorporar los valores y la filosofía que la empresa desea mostrar a su consumidor. $Y$ esto no es nuevo, pues ya se ha dicho que el directivo procura alinear los objetivos del empleado con los objetivos estratégicos de la organización (Zangaro, 2011). Y la capacitación le puede ser útil al directivo en ese propósito. Por ejemplo, se considera que, si el trabajador se apropia de los objetivos organizacionales, "se obtiene una ventaja competitiva que lleva a la organización al éxito" (Participante 13D, comunicación personal, marzo de 2018). Los directivos consideran que la capacitación logra darle valor a la compañía a través del mejoramiento de procesos, derivados de los conocimientos aprendidos. En este sentido, se asegura que "una capacitación efectiva se traduce en mejoras de procedimientos, reducción de tiempos de procesos y crecimiento de la productividad" (Participante 18D, comunicación personal, marzo de 2018).

De esta manera, tanto trabajadores y directivos ven la capacitación como algo que impacta favorablemente el desempeño. Pero para saber esto, es necesario evaluar la capacitación en sus diferentes dimensiones. Sin embargo, los hallazgos del estudio indican que la percepción es medida frecuentemente, pero, los resultados en términos de productividad o de crecimiento personal casi no se miden. Lo particular aquí es que, aunque no se midan esos resultados, trabajadores y directivos tienen la idea de que la capacitación impacta favorablemente el desempeño. Y esta idea es generalizada en las organizaciones abordadas. Pero ¿cuál es la estructura de esta representación? 
La idea que la capacitación impacta favorablemente el desempeño aparece fundamentada (estructura interna) en tres elementos: en primer lugar, los entrevistados sostienen que lo que se aprende se lleva a la práctica y esto mejora sus habilidades. En segundo lugar, consideran que con la capacitación actualizan el conocimiento, y si ese conocimiento se puede aplicar, entonces la capacitación es útil. En tercer lugar, las capacitaciones referidas a relaciones humanas y valores, son consideradas las más enriquecedoras. Ahora bien, como esta idea de resultados individuales producto de las capacitaciones no tiene soporte en mediciones de las organizaciones, podría pensarse que esta representación se ha reforzado discursivamente, mediante la comunicación social, donde se origina principalmente la construcción de las representaciones (Araya (2002).

\section{La oferta de capacitación como indicador del interés en los trabajadores}

Los recursos invertidos por la empresa para capacitar son vistos por los directivos como una evidencia del interés de la empresa hacia los empleados y esa oferta de capacitación está acompañada (primer factor) con el crecimiento de la empresa y del trabajador. Por tanto, puede decirse que se ha configurado la siguiente representación: la oferta del programa de capacitación es un indicador que la compañía se interesa en los trabajadores, sobre esto se explicará más adelante. Un segundo factor en la estructura de esta representación es que la capacitación hace que los empleados se identifiquen y se fidelicen con la empresa, lo que podría incidir en retenerlos y fidelizarlos (Beynon, Jonesm Pickernell y Packham, 2014). Este argumento se observa tanto en los libros de texto, cuando explican las bondades de la capacitación, como en el discurso de los jefes de RH. Un tercer elemento en esta representación es que el trabajador siente que, si es capacitado, va a tener las habilidades para desarrollar sus funciones o para resolver problemas. Pensar que con la capacitación se obtienen esas habilidades lleva a que el trabajador sienta seguridad en sí mismo (Pilati y Borges, 2012), y esa seguridad, a su vez, hace que se sientan apoyados para acoplarse a los cambios laborales. Recordemos que las representaciones, incorporan principios interpretativos y orientadores de las prácticas, a partir de la comprensión, la valoración, la comunicación y la actuación.

Además, para esta población, pensar en capacitación es pensar en la oportunidad de ser mejores personas, y desarrollarse como profesionales. Los directivos declaran que la capacitación permite generar sentido de pertenencia y dar valor a las personas. Lo particular es que, en el discurso de los entrevistados, esa valoración y ese sentido de pertenencia, se suponen, que impacta de alguna manera al desempeño, tesis que no queda evidenciada porque, como se mencionó anteriormente, la mayoría de las organizaciones no miden el impacto de la capacitación en los resultados. Ahora bien, en algunas organizaciones sí se evalúa el sentido de pertenencia, pero no es tan sencillo afirmar que un alto sentido de pertenencia obedezca directamente a la capacitación. 
Por otro lado, en los directivos emergió la idea que, a veces, la capacitación no sirve porque es un gasto, porque se hace por cumplir con la ley o porque sencillamente tiene poco impacto. Por ejemplo, en el primer caso se consideró un gasto porque el retorno económico que esta genera no es tangible. No obstante, los directivos ( $y$ a veces algunos consultores) argumentaron que la capacitación impacta favorablemente la competitividad para la empresa. Pero hay un pero. ¿Cómo afirmar ese impacto sobre la competitividad cuando en muy pocas ocasiones miden el aprendizaje o los resultados?

En el segundo caso, los entrevistados manifestaron que se capacita por cumplir con la ley y que si no existiera la ley no se ofrecerían cursos de capacitación. Así lo expresa un entrevistado:

Hay capacitaciones donde se repite la misma cosa; la persona que prepara el tema no lo prepara bien o simplemente no se actualiza; se hace por llenar el requisito; y ahí uno considera que se perdió el tiempo de esta capacitación... (Participante 15D, comunicación personal, marzo de 2018).

Finalmente, la capacitación es vista como algo negativo cuando se considera mal enfocada, en función de las competencias que se requieren desarrollar y del desempeño organizacional. Así lo sostiene uno de los entrevistados, y anota: "me meten en capacitaciones donde no tengo nada que ver, pues mi perfil no aplica para eso. [...] y uno dice: esta persona no se fijó. Esa capacitación no me sirve"(Participante 12D, comunicación personal, marzo de 2018). Aquí es importante señalar lo siguiente: las representaciones con algún sentido negativo (es un gasto, se hace por cumplir, está mal enfocada) están construidas sobre la base que las capacitaciones no tienen el efecto esperado, es decir, la representación se construye en función del desempeño organizacional. De allí que se crea que las capacitaciones no son buenas si no ayudan a los resultados organizacionales, como se supone que debe suceder, de acuerdo con los libros de texto.

En este punto es importante llamar la atención sobre algo: aun la construcción de esta representación con orientaciones negativas parte de un sinsentido, puesto que no se miden los resultados ni la aplicación de los conocimientos. Con esto no se está diciendo que la construcción de una representación tenga que estar atada a un evento material (u objetivo), como, por ejemplo, los resultados de la medición del impacto de la capacitación. No, es claro que las representaciones se pueden construir sobre bienes inmateriales o intangibles, lo que significa que, sean positivas o negativas las actitudes de la representación están asociadas a un futuro desempeño, o a un desempeño esperado, en todo caso. 
Figura 2. Representaciones de directivos sobre la capacitación.

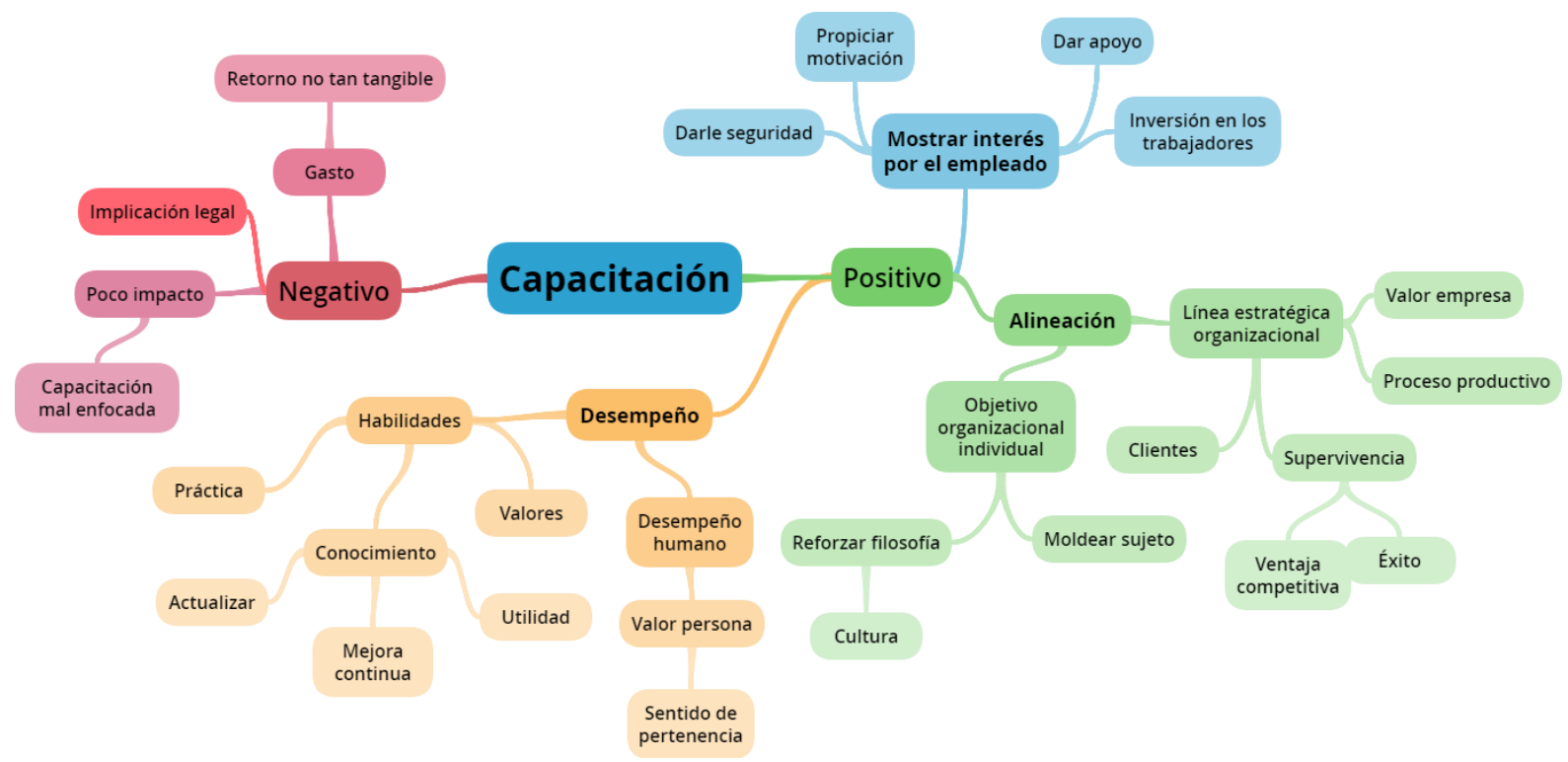

Fuente: Elaboración propia (2019).

\section{Las representaciones de los empleados}

Las representaciones que tienen los trabajadores sobre la capacitación tienen un carácter positivo: contribuyen a la mejora del desempeño, impactan favorablemente la satisfacción laboral y son una muestra de que la empresa los valora.

\section{La mejora en el desempeño}

Los empleados piensan que con la capacitación se generan conocimientos, los cuales les permiten rendir más en sus actividades laborales. Esto se evidencia en las siguientes expresiones: "Gracias a la capacitación puedo desenvolverme muy bien en mis funciones, además todo conocimiento adquirido es muy útil" (Participante 1E, comunicación personal, marzo de 2018). Otro entrevistado afirmó: "lo que he aprendido en la capacitación se ve reflejado en el mejoramiento continuo que he presentado después de la capacitación" (Participante 3E, comunicación personal, marzo de 2018). Por consiguiente, se podría pensar que al haber aprendido algo "útil", el empleado mejora sus habilidades y ese impacto en las habilidades le permite crecer profesionalmente. Pero, ¿cómo es posible que a partir de la adquisición de conocimientos específicos se crezca en la parte profesional? Ante esta pregunta, trabajadores y directivos exponen que la razón es que los empleados mejoran en tres dinámicas de la empresa: el desempeño, el servicio al cliente y la capacidad para la resolución de retos. 
En cuanto a la mejora del desempeño, se puede decir que no se encontraron registros de que la capacitación incremente el desempeño. Sin embargo, los empleados creen que mejoran su desempeño por haber recibido la capacitación: "Gracias a la capacitación de mi empresa siento que puedo manejar los sistemas de una forma mucho más efectiva"(Participante 1E, comunicación personal, marzo de 2018). De acuerdo con la teoría de las representaciones sociales, la anterior expresión lleva a pensar que "el proceso de objetivación se refiere a la transformación de conceptos abstractos extraños en experiencias o materializaciones concretas. Por medio de él lo invisible se convierte en perceptible" (Araya, 2002, p. 35). De allí que el entrevistado afirme: "siento que puedo manejar...de forma más efectiva...".

Por otro lado, en cuanto al servicio al cliente, gracias al estudio realizado por Sánchez, et ál., (2011), se conoce que la satisfacción que siente el cliente con la calidad del servicio, está influenciada fuertemente por la calidad de la capacitación que recibe el empleado. Esto lo refleja un participante, al afirmar que: "de la capacitación depende que podamos trabajar de la mejor manera, podamos tener la mejor actitud; pero si no tenemos el conocimiento, nos sentiríamos inseguros y no haríamos las cosas bien"(Participante 5E, comunicación personal, marzo de 2018).

Por último, respecto a la mejora en la capacidad de resolución de retos, los entrevistados dan a entender que la capacitación, al brindarles nuevas herramientas para el tratamiento de dificultades, les permite reevaluar las situaciones problemáticas y buscar mejores soluciones. Como se ha mencionado, estudiar una representación social implica determinar qué se sabe, qué se cree, cómo se interpreta y cómo se actúa. Por tanto, en este caso, la lógica parece ser: cómo siento que tengo las capacidades, entonces me arriesgo a afrontar nuevos retos que podrán resolverse de manera exitosa.

Para los directivos, la capacitación permite alinear la mentalidad del empleado hacia los objetivos de la empresa, y los empleados perciben que la capacitación es el móvil para enfocar sus actividades en el logro de los objetivos organizacionales. Como se señaló anteriormente, los elementos estructurantes de una representación no solamente pueden estar imbricados entre sí, sino que, además, elementos que hacen parte de una representación en directivos, también pueden estar imbricados con elementos de una representación de los empleados (aunque pertenezcan a organizaciones diferentes). Informando al empleado sobre los planes estratégicos se busca moldear al sujeto para que se apropie de los principios establecidos y actúe de acuerdo a ellos. Esta búsqueda va de la mano con la necesidad que tiene la organización de controlar al empleado (Alvesson y Willmott, 2013) y, de acuerdo con los entrevistados, cambiar su mentalidad en pro de la organización. Al respecto, un participante (7E, comunicación personal, marzo de 2018) menciona que: "cuando uno recibe capacitación lo primero que percibe es que la empresa está interesada en desarrollar integralmente a los empleados. Cuando se percibe eso, se propicia un ambiente de trabajo muy positivo, se genera sentido de pertenencia en los empleados porque están recibiendo beneficios adicionales". 
Parece sencilla la asociación: como la oferta de capacitación da la sensación de que los empleados son importantes, de esta manera logra, según los entrevistados, fidelizar al empleado y fortalecer así la estrategia de la organización en el tiempo. Con esta investigación no sabemos si la capacitación dispara el rendimiento individual o fideliza a los trabajadores. Pero, al menos, sabemos que existe esa creencia.

Figura 3. Comparación de representaciones sobre desempeño

\section{Directivos}

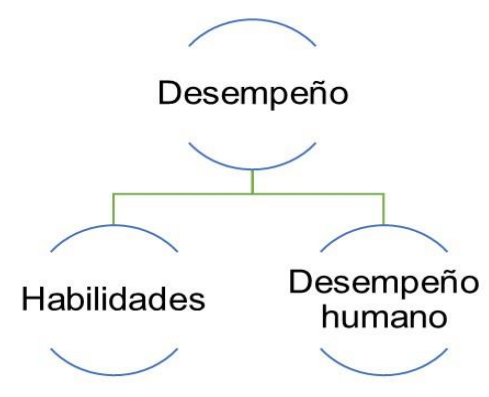

\section{Empleados}

Conocimiento que mejora el desempeño

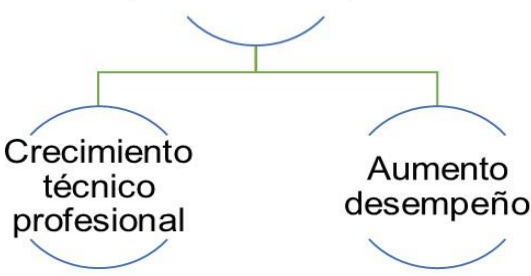

Fuente: Elaboración propia (2019).

\section{Los efectos sobre la satisfacción laboral}

¿Cómo es posible que por medio de la capacitación se logre la satisfacción laboral? La cuestión parece radicar en este esquema: primero se dictan varias sesiones, teóricas o prácticas y luego se espera que el trabajador utilice los nuevos conocimientos y habilidades en el área de trabajo. Después, cuando el trabajador termina la capacitación y empieza a aplicar los nuevos conocimientos en su trabajo y en su vida, él considera que esa aplicación produce un cambio positivo en su conducta, lo que hace que se sienta satisfecho. Al respecto, un empleado comenta que sintió que la capacitación tuvo un impacto muy grande en su vida. Empezó a ponerse metas más ambiciosas y cada vez que terminaba un proyecto, utilizando lo aprendido, se sentía muy satisfecho. Esa es entonces la secuencia lógica que genera satisfacción laboral gracias a la capacitación. Ese es el esquema, al menos en palabras de los entrevistados. En la vida cotidiana, Materán (2008) sostiene que las circunstancias y las relaciones sociales exigen del grupo social que estos estén en situación de responder ante ciertas circunstancias. No se está negando que exista asociación entre estos conceptos. Cabe resaltar que, aunque pueda existir una vinculación entre capacitación y satisfacción laboral, esta requiere otros aspectos como reconocimiento, reglas eficaces, respeto, salarios justos (Bercu, 2017). De todas formas, los empleados sienten que tienen herramientas para alcanzar la satisfacción laboral, y para ellos es una realidad. Veamos otro ejemplo: "Cuando hay una persona capacitada es menos probable que se cometa un error que pueda llevar a algo más grave", afirma el participante 17E, (comunicación personal, marzo de 2018). 
En sectores como la construcción y maquinaria pesada, la capacitación se presenta como de vital importancia para garantizar la supervivencia del trabajador; una vez el empleado capacitado está en su puesto de trabajo, tiene el conocimiento de los pasos para realizar adecuadamente su labor, y este saber explicaría parte de la seguridad que siente en la toma de decisiones. En este caso, se puede decir que la transformación de un concepto en una imagen pierde su carácter simbólico arbitrario y se convierte en una realidad con existencia autónoma (Araya, 2002). El anclaje consiste, precisamente, en transformar lo que es extraño en familiar, o sea hacer inteligible lo que no es familiar.

\section{La empresa nos capacita porque nos valora como empleado}

Una de las representaciones que emerge a lo largo de la investigación se refiere a la valoración del empleado. Para los trabajadores, la capacitación es una muestra de que la empresa los valora. Y esto, se materializa en el crecimiento personal y la conciencia en sí mismo. Los trabajadores hablan de crecimiento en valores, aptitudes y habilidades gracias a la capacitación. Para ellos, esas habilidades permiten aumentar el desempeño, incluso sostienen que cambian su ámbito familiar y su comportamiento en la sociedad. Lo anterior "se logra cuando el empleado adquiere y al mismo tiempo actualiza su conocimiento" (Participante 13E, comunicación personal, marzo de 2018). Al parecer la idea de adquirir conocimiento es muy importante para las personas y este se adquiere gracias a la capacitación.

Así entonces, ante la pregunta inicial de si la capacitación es vista como un beneficio que reciben los trabajadores, los hallazgos sugieren que sí. Y dicho sí se obtiene por una de las siguientes vías. Primero, se da por sentado de que la capacitación mejora los resultados operacionales (directivos y trabajadores creen que sí); y el contenido de una representación tiene que ver con una forma particular de conocimiento, con un universo de creencias; pero, dado que los resultados no se miden, entonces, la otra vía es el llamado crecimiento personal. La idea que la capacitación es un beneficio que se recibe se soporta en expresiones como: "todo lo que uno aprende, le sirve para la vida" o "con la capacitación siempre hay un crecimiento personal". Expresiones de trabajadores que inclusive no han recibido capacitación. Por consiguiente, lo aprendido que sirve para la vida y para el crecimiento personal es gracias a la capacitación, de allí que esta sea vista como un beneficio que recibimos de la organización. 
Figura 4. Comparación de representaciones sobre interés hacia el empleado

\section{Directivos}

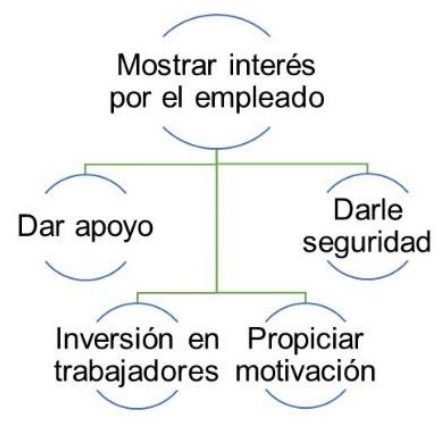

Empleados

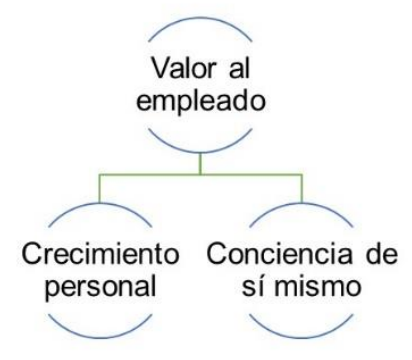

Fuente: Elaboración propia (2019).

Por otro lado, se considera que los sistemas de interpretación proporcionados por la representación guían la conducta (Araya 2002, p. 37). Dicho vínculo representación-conducta se puede observar en el hecho de que algunos trabajadores consideran que (además de ser un beneficio) con la capacitación se sienten en capacidad de enseñar a los demás lo que han aprendido. Los trabajadores afirman que cuando están constantemente capacitados tienden a "tomar conciencia de sí mismos, es decir, a transformar su mentalidad. Al darse esto, también cambia sus actitudes" (Participante 16E, comunicación personal, marzo de 2018). Esto indica que la capacitación no solo afecta la parte técnica sino también el comportamiento, o al menos la actitud, cuando expresan sentirse en capacidad de enseñar a otros. Y ello daría cuenta del vínculo comportamiento-representación, es decir, en términos de Abric (2001), parece que la práctica capacitación genera un desplazamiento en la representación que el sujeto tiene de sí mismo. 
Figura 5. Representaciones de los empleados sobre la capacitación

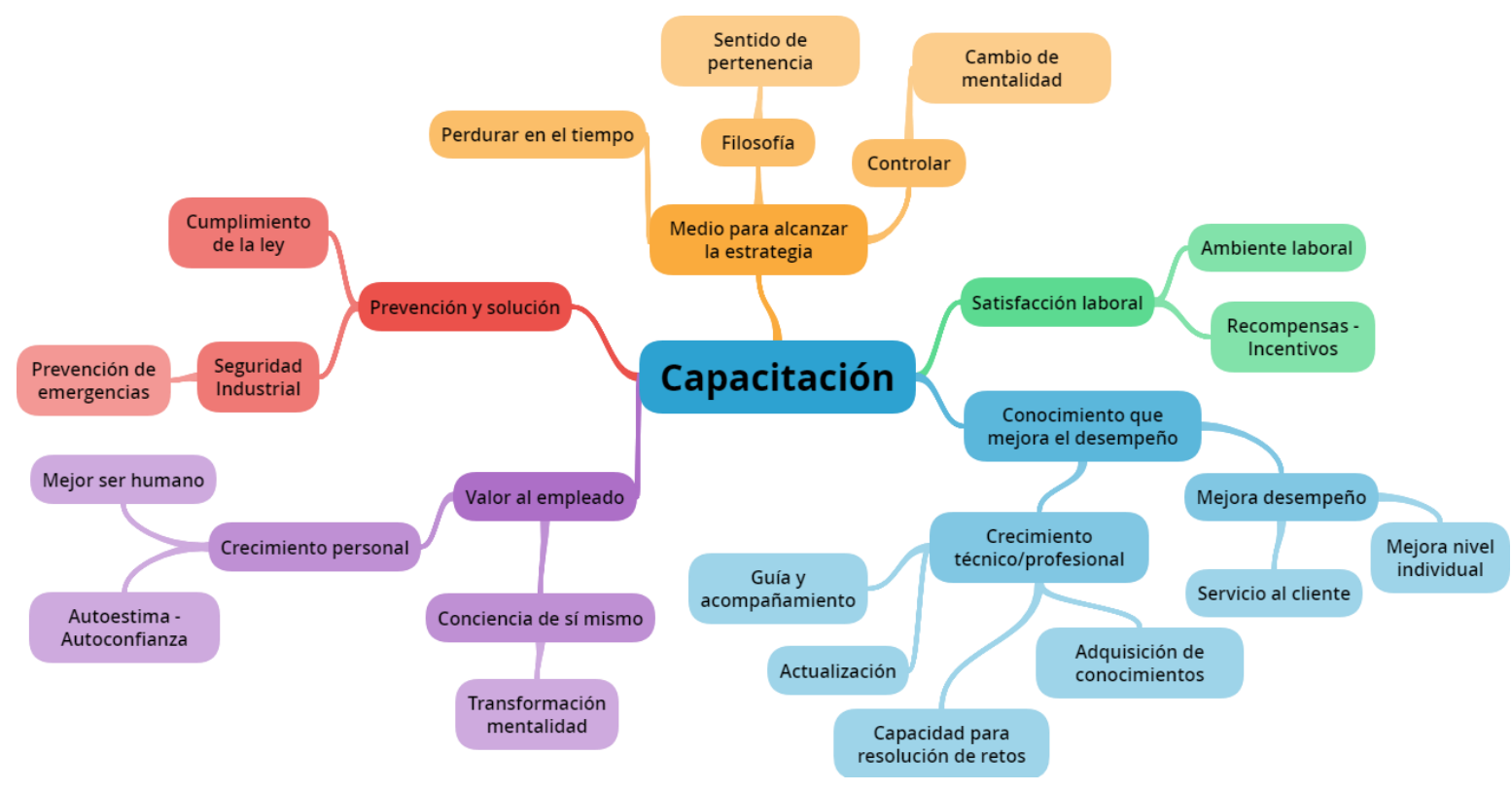

Fuente: Elaboración propia (2019).

\section{Conclusiones}

Los hallazgos encontrados muestran que sin importar el rol que se tenga en una empresa (directivo o empleado), se tienen ideas comunes acerca de los procesos de capacitación, es decir, se construyen representaciones sociales similares.

Al comparar las representaciones sobre la capacitación con la literatura, lo primero que se encuentra es que los textos no hacen mucho énfasis en los aspectos negativos de la capacitación, simplemente se alude a que la capacitación permite lograr cosas positivas. Este estudio señala que cuando la capacitación se considera que está mal enfocada se genera una idea negativa de ella. Ahora bien, estar mal enfocada quiere decir que no es útil para la productividad. No obstante, esta lógica genera dificultad cuando se retoman las fases de evaluación de la capacitación (percepción, aprendizaje, aplicación y resultados); lo común es que las empresas evalúen las dos primeras fases, pero no se evalúa la aplicación de conocimientos ni los resultados de la capacitación en términos de desempeño. Lo que sugiere que esa idea de la capacitación, a veces, está mal enfocada, es tan solo una idea intuitiva. Adicionalmente, aunque los textos hablen de crecimiento personal, lo hacen con visos funcionalistas pues en ellos la capacitación es considerada mal enfocada cuando no es útil para la productividad. 
Por otra parte, se puede afirmar que se ha construido la representación, en trabajadores y directivos, que la capacitación es una manera de darle valor al empleado, sin embargo, los elementos que sustentan dicha representación varían de un grupo a otro. Para los directivos es algo que la empresa les da a los empleados para proporcionarles seguridad, apoyo o satisfacción. Pero, sobre todo, la sola existencia de la posibilidad de capacitarse es una manera de mostrar que la empresa sí valora a los trabajadores. Se puede decir que solo la existencia porque no todos los trabajadores participantes han recibido capacitación y aun así manifiestan los beneficios de esta.

Para los empleados el valor está dado por la consecuencia de la capacitación, ya sea en habilidades técnicas o en crecimiento personal (no medido), pero también, por la satisfacción laboral o conocimiento que sirve para el desempeño o para la vida. En otras palabras, los resultados muestran que se ha construido la representación en que la capacitación es "algo" que el trabajador "recibe" de la empresa, con el mensaje que él es importante para esta.

Por otro lado, dado que no hay mediciones de la aplicación de lo aprendido ni de los resultados (operacionales), refuerza el hecho que se ha construido la idea en los trabajadores de la sola existencia de la capacitación es un "indicador" que ellos son importantes para la empresa.

Finalmente, tanto directivos como empleados estuvieron de acuerdo en que aspectos como el desempeño, el sentido de pertenencia para con la empresa, las habilidades técnicas y profesionales, y el crecimiento personal, después de las capacitaciones tiene efectos positivos. Aunque esta "mejora" de la que ellos hablan no sea evaluada en ninguna de las empresas abordadas en esta investigación, sí es ampliamente difundida por los libros de texto que utilizan las empresas para educar a los directivos en el tema de la capacitación.

\section{Referencias}

Abric, J. (2001). Pratiques sociales y Représentations (1 $1^{\mathrm{a}}$ ed.). [Traducido por Flora Palacios] México: Ediciones Coyoacán S. A.

Alvesson, M. (2015). Organizational Culture and work. In Edgell, S., Gottfried, H. \& Granter, E. (Eds). The SAGE Handbook of the Sociology of Work and Employment (pp. 262-281). Suecia: SAGE Publications Ltda. http://dx.doi.org/10.4135/9781473915206.n15

Alvesson, M., \& Willmott, H. (2013). Making sense of management: a critical introduction. Action Learning Research and Practice, 10(3), 287-290. https://ideas.repec.org/a/taf/alresp/v10y2013i3p287290.html 
Alvesson, M., \& Willmott, H. (2014). Studying Management Critically (1 ${ }^{\text {a }}$ ed.). Londres: SAGE Publications Ltda. http://sk.sagepub.com/books/studying-management-critically/n5.xml

Araya, S. (2002). Las representaciones sociales: Ejes teóricos para su discusión. (19a ed). Costa Rica: Facultad Latinoamericana de Ciencias Sociales (FLACSO).

Bercu, A. (2017). Impact of employees' training programmes on job satisfaction. Current Science, 112(7), 1340-1345. https://www.currentscience.ac.in/php/toc.php?vol=112\&issue=07

Beynon, M., Jones, P., Pickernell, D., \& Packham, G. (2014). Investigating the impact of training influence on employee retention in small and medium enterprises: A regression-type classification and ranking believe simplex analysis on sparse data. Expert Systems, 32(1), 141-154. https://doi.org/10.1111/exsy.12067

Burbano, J. (2016). Discursos y prácticas de selección en los manuales de Gestión Humana. En Carvajal, L., \& López, O. (Edits.). Selección de personal: Discursos, prácticas, tecnologías (1a ed.). (p.51-70). Bogotá: Editorial Pontificia Universidad Javeriana.

Chiavenato, I. (2009). Gestión del talento humano (3a ed.). (Traducido por P. M. Sacristán) México: McGraw Hill.

Dessler, G. (2009). Administración de Recursos Humanos (11 a ed.). México: Pearson Education, Inc.

Dolan, S., Valle, R., Jackson, S., \& Schuler, R. (2007). La Gestión de Recursos Humanos (3a ed.). Madrid: Mc Graw Hill.

Esquivei, R., Félix, G., \& Bello, R. (2014). Evaluación del impacto de la capacitación con lógica difusa. Ingeniare, 22(1), 41-52. http://dx.doi.org/10.4067/S0718-33052014000100005

Fawad, K. (2012). An integrated model of training effectiveness and satisfaction with employee development interventions. Industrial and Commercial Training, 44(4), 211-222. https://doi.org/10.1108/00197851211231487

Flouris, T., \& Kucuk, A. (2017). Linkages between Risk and Human Resources Management in Aviation: An Empirical Investigation and the Way Forward in Selection of Ideal Airport Manager. In Yilmaz, A \& Flouris, T. Corporate Risk Management for International Business. (pp. 141-151). Switzerland: Springer Singapore. https://doi.org/10.1007/978-981-10-4266-9

Gil, A., \& Mataveli , M. (2016). Rewards for continuous training: a learning organisation perspective. Industrial and Commercial Training, 48(5), 257-264. https://doi.org/10.1108/ICT-11-2015-0076 
Guber, R. (2001). La entrevista etnográfica o el arte de la "no directividad". En Guber, R. La etnografía: Método, campo y reflexibilidad (p. 75-100). Bogotá, Colombia: Grupo Editorial, Norma.

Jaraba, R. (2012). Modulo Psicología del aprendizaje Unidad I. Sincelejo: Corporacíon Universitaria del Caribe (CECAR). https://docplayer.es/23216655-Psicologia-del-aprendizaje.html

Jodelet, D. (1986). La representación social: fenómenos, conceptos y teoría. En Moscovici, S. (Ed.), Psicología Social II: Pensamiento y vida social (pp. 469-494). Barcelona: Páidos.

Kooij, D., \& Boon, C. (2018). Perceptions of HR practices, person-organisation fit, and affective commitment: The moderating role of career stage. Human Resource management journal, 28(1)6175. https://doi.org/10.1111/1748-8583.12164

Landini, F., Bianqui, V., \& Russo, M. (2013). Evaluación de un proceso de capacitación para extensionistas rurales implementado en Paraguay. Revista de Economia e Sociologia Rural, 51(1), 9-30. http://dx.doi.org/10.1590/S0103-20032013000600001.

Materán, A. (2008). Las representaciones sociales: un referente teórico para la investigación educativa. Geoenseñanza, 13(2), 243-248. http://www.saber.ula.ve/handle/123456789/28953

Mazur, R., \& Woodland, R. (2017). Evaluation of a Cross-Cultural Training Program for Pakistani Educators: Lessons Learned and Implications for Program Planning. Evaluation and Program Planning, 62, 25-34. https://doi.org/10.1016/j.evalprogplan.2017.02.011

Mohamed, F., \& Mustafa, M. (2018). The Effect of Training and Development towards Employee Performance: A Case Study in Proton Tanjung Malim. Global Business and Management Research: An International Journal, 10(3), 777-781. http://www.gbmrjournal.com/vol10no3.htm

Morin, M. (2016). Jean-Claude Abric: La Pratique Sociale au Cœur de la Recherche. Papers on Social Representations, 25(2), 11.1-11.9. http://psr.iscte-iul.pt/index.php/PSR/article/view/50

Moscovici, S. (1961). El psicoanálisis, su imagen y su público. Buenos Aires: Huemul.

O'Connor, J., \& Little, B. (2012). Evaluation-training's ignored leverage point. Industrial and Commercial Training, 44(5), 273-280. https://doi.org/10.1108/00197851211245022

Pilati, R., \& Borges, J. (2012). Training Effectiveness: Transfer Strategies, Perception of Support and Worker Commitment as Predictors. Psicología del Trabajo y de las Organizaciones, 28(1), 25-35. http://dx.doi.org/10.5093/tr2012a3 
Raigosa, D., \& Marin, B. (2010). Efficacy beliefs training: a proposal to reduce burnout and improve levels of engagement among employees. International Journal of Psychological Research, 3(2), 86-92. https://doi.org/10.21500/20112084.815

Rueda, C., Jiménez, K., y Sánchez, Y. (2015). Percepciones del impacto de la capacitación, compensación y selección del personal en la eficiencia de los proyectos. AD-MINISTER (27), 5-26. http://dx.doi.org/10.17230/ad-minister.27.1.

Sánchez, R., Arce, B., Ramírez, J., \& Hernández, S. (2011). La capacitación como predictora de satisfacción del cliente en organizaciones turísticas. Ingeniería Industrial, XXXII(3), 207-212. https://www.redalyc.org/articulo.oa?id=3604/360433577006

Shaheen, F., Ghayyur, M., \& Yasmeen, G. (2014). An Investigation of Training and Development Programs on Employees Satisfaction: A Case Study of Marks and Spencer London, UK. Putaj Humanities \& Social Sciences, 21(1), 133-142.

Tito, P. (2003). El trabajador es el recurso más valioso de que diponen las empresas. Gestión en el Tercer Milenio. 6(12), 29-33. https://doi.org/10.15381/gtm.v6i12.9841

Vutâ, D., \& Fârcas, A. (2015). The Role of Training in Organizational an Employee Development. Revista

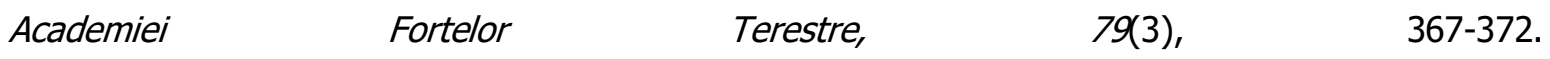
http://www.armyacademy.ro/reviste/rev3_2015/VUTA.pdf

Young, S., \& Nam, J. (2018). Effects of training and development on employee outcomes and firm innovative performance: Moderating roles of voluntary participation and evaluation. Human resource management, 57(6), 1339-1353. https://doi.org/10.1002/hrm.21909

Zangaro, M. (2011). Subjetividad y trabajo: el management como dispositivo de gobierno.Trabajo $y$ Sociedad, XV(16), 163-177. https://www.unse.edu.ar/trabajoysociedad/\#N\%C3\%BAmero_16 